Autor: Francisco Herlânio Costa Carvalho

Orientador: Prof. Dr. Antonio Fernandes Moron

Dissertação de Mestrado apresentada ao Departamento de obstetrícia da Universidade Federal de São Paulo Escola Paulista de Medicina em 19 de março de 2001.

Nos últimos anos, investigações sobre o sistema vascular venoso tornaram-se cada vez mais importantes na avaliação da função miocárdica fetal. O objetivo deste estudo foi estabelecer valores de referência para a velocidade do fluxo sanguíneo no duto venoso durante as diferentes fases do ciclo cardíaco e para os vários índices descritos na literatura. Doppler pulsátil e colorido foram utilizados neste estudo transversal e prospectivo que examinou 276 pacientes entre 10 e 14 semanas de gestação. Foram excluídos da análise os fetos com translucência nucal aumentada, malformações congênitas, anomalias cromossômicas, parto prematuro, óbito intraútero ou fenótipo anormal ao nascimento. O melhor plano para visibilizar o duto venoso foi o médio sagital do abdome fetal. Curvas de referências foram construídas para todos os parâmetros analisados. Somente observamos aumento na velocidade duran- te contração ventricular (onda S) e durante diástole ventricular precoce (onda D). As velocidades $S-D$ e A, bem como todos os indices estudados permaneceram constantes ao longo das idades gestacionais analisadas. A ausência de modificações importantes na onda de velocidade de fluxo no duto venoso devese ao fato de que a redução da resistência placentária e a maturação na função diastólica ventricular (aumento na complacência) só ocorrem após o período estudado. Os valores de referência estabelecidos por esse estudo podem servir como base para acompanhamento de gestações normais, para diagnóstico de insuficiência miocárdica fetal ou rastreamento de anomalias cromossômicas.

Palavras-chave: Gravidez normal. Dopplervelocimetria. Duto Venoso.

RBCO 23(6):403,2001

Resumo de Tese

\title{
Biópsia de Miométrio por Histeroscopia no Diagnóstico da Adenomiose
}

Autor: Walter Antônio Prata Pace

Orientadora: Prof. ${ }^{a}$ Dr. ${ }^{a}$ Maria do Carmo Borges de Souza

Tese apresentada ao Instituto de Ginecologia da Universidade Federal do Rio de Janeiro como parte dos requisitos necessários para obtenção do título de Doutor em ciências, em 4 de dezembro de 2000.

Objetivo: estabelecer o valor da biopsia de miométrio por histeroscopia para detecção da adenomiose.

Métodos: foram selecionadas 30 pacientes (idade 28 à 54 anos), clientes de clínica privada no período de 3 de março de 1997 à 21 de outubro de 1999, com o diagnóstico clínico e ultra-sonográfico sugestivo de adenomiose (com prole definida); todas elas tinham indicação de histerectomia. As histerectomias foram realizadas em centros cirúrgicos precedidos de biopsia do miométrio por histeroscopia, sendo obtidos as amostras na parede posterior, próximo ao fundo.

Resultados: na análise dos 30 casos a biopsia de miométrio foi confrontada com a peça cirúrgica (útero) como padrão-ouro mostrou sensibilidade de $82 \%$, especificidade de $50 \%$, valor preditivo positivo de $95 \%$, valor preditivo negativo de $16 \%$, falso-positivo de $50 \%$, falso-negativo de $17 \%$.

Conclusões: embora os testes estatísticos não tenham sido significativos nesta casuística, a técnica mostrou-se factivel e segura, capaz de fornecer material adequado para estudo.

Palavras-chave: Histerectomia. Histeroscopia. Adenomiose. Endometriose. 


\section{Repercussões Maternas, Reprodutivas e Perinatais do Tratamento com Extrato Aquoso de Folhas de Bauhinia forficata (Pata-de-vaca) na Prenhez de Ratas Não-diabéticas e Diabéticas}

Autor: Gustavo Tadeu Volpato

Orientadora: Profa. Dra. Iracema de Mattos Paranhos Calderon

Dissertação de Mestrado apresentada ao Departamente de Ginecologia e Obstetrícia - Faculdade de Medicina de Botucatu/Unesp, em 23 de março de 2001.

Introdução: a despeito da ação, comprovada ou não, é comum a constatação de que gestantes diabéticas fazem uso de plantas popularmente conhecidas como hipoglicemiantes. Uma das mais citadas é a Bauhinia forficata (pata-de-vaca). O risco está em não se conhecer seus efeitos e possiveis repercussões materno-fetais.

Objetivo: Avaliar os efeitos do extrato aquoso de folhas de $B$. forficata sobre o metabolismo materno, a performance reprodutiva, o desenvolvimento fetal e a teratogênese na prenhez de ratas diabéticas e nãodiabéticas.

Material e Método: Ratas Wistar prenhes constituíram quatro grupos: não-diabético e diabético controles (não-tratados); não-diabético e diabético tratados com extrato aquoso de folhas de B. forficata. O diabete foi induzido antes da prenhez, pela injeção intravenosa de estreptozotocina (40 mg/kg de peso). A administração do extrato da planta ou da água destilada (grupos controle) foi por via oral em doses crescentes: $500 \mathrm{mg} / \mathrm{kg}$ do 0 ao $4^{\circ}$ dia de prenhez, 600 $\mathrm{mg} / \mathrm{kg}\left(5^{\circ}\right.$ ao $\left.14^{\circ}\right)$ e $1000 \mathrm{mg} / \mathrm{kg}\left(15^{\circ}\right.$ ao $\left.20^{\circ}\right)$, ajusta- das diariamente conforme o peso do animal, sendo administrados dois terços da dose pela manhã e um terço à tarde. Durante a prenhez foram avaliados o ganho de peso e os niveis glicêmicos maternos e no $21^{\circ}$ dia foi realizada cesárea, com avaliação do peso dos recém-nascidos e das placentas, da performance reprodutiva e da freqüência de anomalias e malformações fetais.

Resultados e Conclusões: Nos grupos de ratas nãodiabéticas, o tratamento com o extrato de $B$. forficata não interferiu na glicemia, no ganho de peso ou na performance reprodutiva materna. Não alterou, também, o desenvolvimento fetal e placentário e não foi teratogênico. Nos animais diabéticos tratados com o extrato das folhas de B. forficata, apesar do nãocontrole da glicemia materna, observou-se queda na freqüência de algumas anomalias esqueléticas e malformações viscerais, quando se comparou com os grupos diabéticos não-tratados.

Palavras-chave: Plantas medicinais. Diabete melito. Malformações. Estudos experimentais.

\section{Efeito da Noretisterona sobre o Índice de Pulsatilidade das Artérias Uterinas de Mulheres na Pós- menopausa em Estrogenioterapia}

Autor: João Pedro Junqueira Caetano Orientador: Prof. Dr. Andy Petroianu

Tese apresentada ao Programa de Pós-Graduação em Cirurgia da Faculdade de Medicina da Universidade Federal de Minas Gerais, como requisito à obtenção do título de Doutor em Medicina, em 20 de dezembro de 2000.

$\mathrm{Na}$ atualidade existem evidências sobre a capacidade vasodilatadora dos estrogênios e dos efeitos benéficos sobre a diminuição dos riscos cardiovasculares em mulheres na pós-menopausa. Quando a paciente apresenta útero, existe a necessidade de se utilizar um progestagênio para contrabalançar os efeitos mitóticos dos estrogênios sobre o endométrio. Um estudo clínico do tipo duplo-desconhecido aleatório foi realizado, no periodo de fevereiro a julho de 1997 com 64 pacientes pós-menopausadas, tendo o objetivo de estudar os efeitos da adição da noretisterona sobre o Índice de Pulsatilidade das artérias uterinas de mulheres na pósmenopausa utilizando estrogênioterapia por via transdérmica (17ß-estradiol). Após a realização do estudo, as pacientes foram divididas aleatoriamente em dois grupos de pacientes da seguinte forma: Grupo I ensaio, com 28 pacientes que receberam $17 \beta$-estradiol e $17 \beta$-estradiol/noretisterona através da via transdérmica; e Grupo II - controle - com 30 pacientes, que receberam placebo por via transdérmica. Não houve diferença entre os grupos no que diz respeito à idade, tempo de menopausa e idade da menopausa. A média do Î́ndice de Pulsatilidade (IP) no Grupo I - ensaio antes do tratamento foi de $3,38 \pm 0,82$; durante a utilização somente de estradiol foi de $2,31 \pm 0,66$; e durante a fase estro-progestagênica foi de 2,34 $\pm 0,63$. 
Comparando o Índice de Pulsatilidade antes e após a estrogenioterapia, a diferença foi de 1,04 $\pm 0,82$, apresentando uma diferença significativa $(p=0,0000)$ e representando uma queda de 30,5 \% nos valores médios do IP antes do tratamento. Quando se associou a noretisterona ao esquema de reposição hormonal, o valor da média dos Îndices de Pulsatilidade não apresentou diferença significativa $(p=0,8075)$. No grupo controle não houve diferença estatística entre os três momentos do estudo ( $p=0,6740$ e $p=0,7520)$. Concluímos que a adição da noretisterona não modificou a diminuição do IP decorrente do $17 \beta$-estradiol por via transdérmica, em mulheres na pós-menopausa.

Palavras-chave: Terapia de reposição hormonal. Menopausa. Ultra-sonografia. Dopplervelocimetria. Climatério.

\section{RBCO 23(6):405,2001}

Resumo de Tese

\section{Liquido Amniótico: Perfil Bioquímico do Desenvolvimento Renal e Fetal}

Autor: Fernando Rocha de Oliveira

Orientador: Prof. Dr. José Antônio Magalhães

Dissertação de Mestrado apresentada ao Departamento de Ginecologia e Obstetrícia e Departamento de Medicina Interna-Serviço de Nefrologia do Hospital das Clínicas de Porto Alegre, Universidade Federal do Rio Grande do Sul, em 24 de abril de 2001.

Base Teórica: a creatinina vem ao longo dos anos representando a função e maturação dos rins fetais. Há necessidade de determinar outros marcadores que possam auxiliar no diagnóstico de distúrbios renais. Objetivos: determinar um perfil e correlacionar marcadores bioquímicos que avaliam função e maturação renal do feto humano no liquido amniótico durante a evolução da gestação normal. Avaliar distribuição de valores para creatinina, N-acetyl-ß-Dglucosaminidase (NAG), $\aleph_{2}$-microglobulina, glicose, uréia, sódio, potássio, cloro, fósforo, cálcio, ácido úrico, albumina e osmolalidade em três períodos gestacionais. Delineamento: estudo transversal avaliou 115 amostras de líquido amniótico em três diferentes períodos gestacionais que consistiam da $13^{\mathrm{a}}$ a $20^{\mathrm{a}}$ semana, $27^{\mathrm{a}}$ a $34^{a}$ e da $36^{a}$ a $42^{a}$ semana. As coletas foram realizadas no Hospital de Clínicas de Porto Alegre.

Resultados: valores da creatinina, NAG, uréia, potássio e ácido úrico apresentaram perfil de crescimento ao longo da gestação $(p<0,05)$. $\$ 2$-microglobulina, glicose, sódio, fósforo, cálcio, albumina e osmolalidade, ao contrário, apresentaram perfil de decréscimo $(\mathrm{p}<0,05)$. §2-microglobulina, glicose e ácido úrico apresentaram significativas correlações $(r>0,6)(p<0,05)$ com a idade gestacional e a creatinina, respectivamente. Uréia, potássio e fósforo apresentaram leves correlações $(r>0,5)(p<0,05)$ com ambas. NAG, sódio, cloro, albumina e osmolalidade não demonstraram significativas correlações $(\mathrm{r}<0,5)(\mathrm{p}<0,05)$.

Conclusões: A creatinina confirmou seu papel de boa correlação com a idade gestacional. §2-microglobulina, glicose e ácido úrico apresentaram bons resultados como marcadores de função e maturação renal. Uréia, potássio e fósforo são possiveis marcadores. NAG, sódio, cloro, albumina e osmolalidade não se mostraram úteis na avaliação do desenvolvimento renal.

Palavras Chave: Gravidez normal. Líquido amniótico. Crescimento fetal.

Expressão do Antigeno Nuclear de Proliferação Celular no Epitélio de Fibroadenoma Mamário de Mulheres Tratadas com Contraceptivo Hormonal Oral Combinado

Autor: Angela Seixas Hueb

Orientador: Prof. Dr. Afonso Celso Pinto Nazário

Tese apresentada à Universidade Federal de São Paulo - Escola Paulista de Medicina para obtenção do Título de Mestre em Ginecologia, em 27 de novembro de 2000.

$\mathrm{O}$ efeito dos anticoncepcionais hormonais orais (ACO) no fibroadenoma é ainda tema controverso. Os poucos trabalhos referentes à cinética celular no epitélio do fibroadenoma durante o uso de ACO são conflitantes. 
Por esta razão e, pelo fato dos ACO serem largamente utilizados, propusemo-nos avaliar a proliferação do epitélio do fibroadenoma por meio da expressão do antígeno nuclear de proliferação celular (PCNA) na vigência de dois ciclos consecutivos de utilização da associação de $0,15 \mathrm{mg}$ de levonorgestrel e 0,03mg de etinilestradiol, em comparação a mulheres com a mesma afecção, na segunda fase do ciclo menstrual. Obtivemos os espécimes por meio de biópsia mamária entre $22^{\circ}$ e o $25^{\circ}$ dia do ciclo, de acordo com a data que antecedeu e sucedeu a menstruação no grupo controle, e o dia da pílula, no grupo estudo. Também foi dosada a progesterona sérica no momento do ato cirúrgico, para bem caracterizar a fase lútea. Os grupos fo- ram pareados para as seguintes variáveis: idade, número de gestações, paridade, menarca e amamentação. Foram utilizados o teste de Levene, Qui-quadrado e teste $\mathrm{t}$ para o estudo estatístico. No grupo estudo, a média de expressão do PCNA foi de $66,24 \%$ e, no grupo controle, de $77,89 \%(p=0,24)$. Nossos resultados demonstraram não haver diferença estatística na expressão do antígeno nuclear de proliferação celular entre as mulheres que tomaram ou não ACO, na segunda fase do ciclo.

Palavras Chaves: Mama: doenças benignas. Fibroadenoma. Contracepção hormonal oral. Proliferação celular.

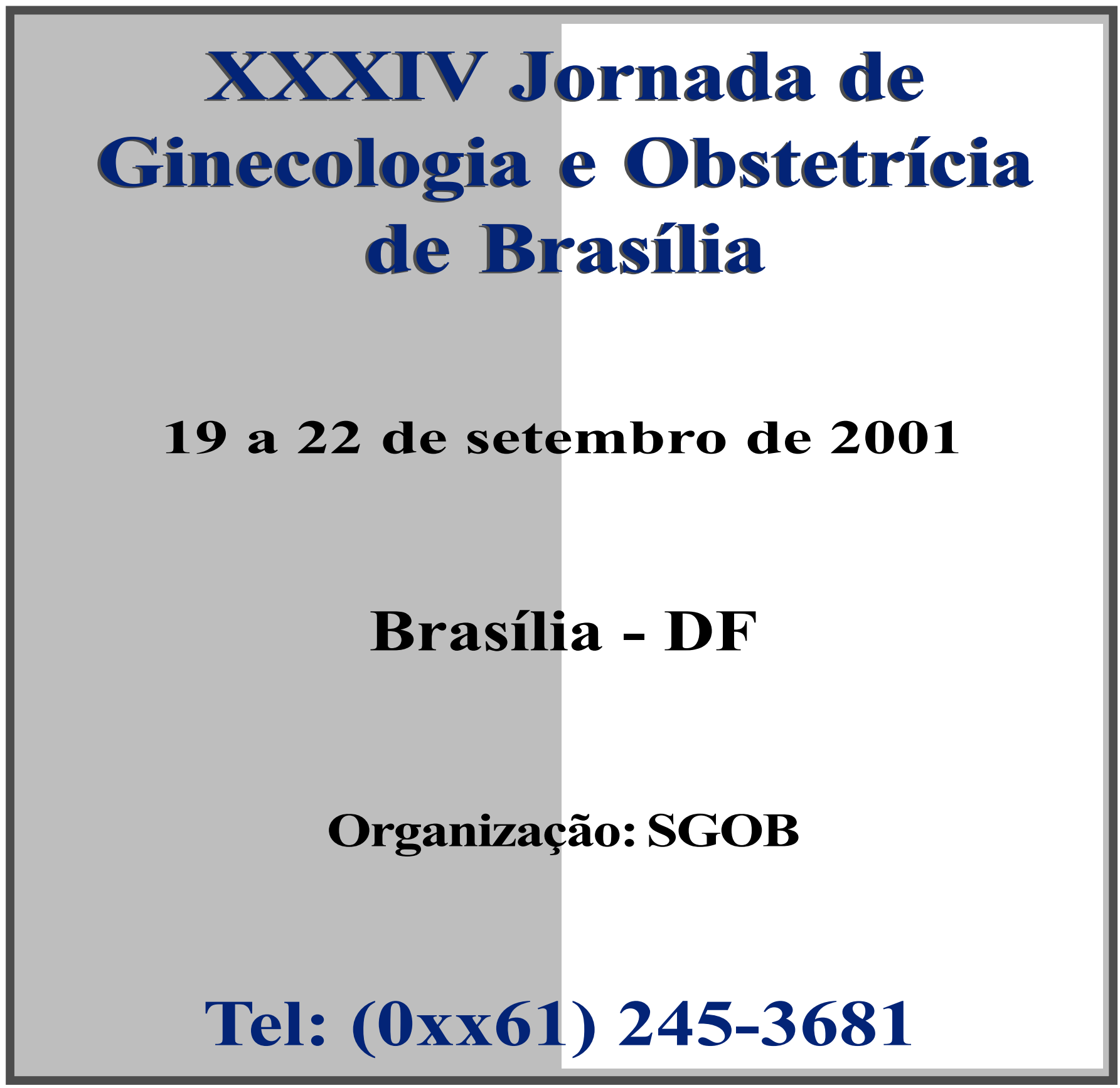

